\title{
Autophagy-associated proteins BAG3 and p62 in testicular cancer
}

\author{
GEORG BARTSCH $^{1 *}$, LUKAS JENNEWEIN $^{2 *}$, PATRICK N. HARTER ${ }^{2,3}$, PATRICK ANTONIETTI $^{4}$, \\ ROMAN A. BLAHETA ${ }^{1}$, HANS-MICHAEL KVASNICKA ${ }^{3,5}$, DONAT KÖGEL ${ }^{3,4}$, \\ AXEL HAFERKAMP $^{1}$, MICHEL MITTELBRONN ${ }^{2,3}$ and JENS MANI ${ }^{1}$ \\ ${ }^{1}$ Department of Urology and ${ }^{2}$ Neurological Institute (Edinger Institute), Goethe University Frankfurt/Main; \\ ${ }^{3}$ German Cancer Consortium (DKTK) and German Consortium for Translational Cancer Research (DKTK), Heidelberg; \\ ${ }^{4}$ Experimental Neurosurgery and ${ }^{5}$ Senckenberg Institute of Pathology, Goethe University of Frankfurt/Main, Germany
}

Received September 15, 2015; Accepted November 3, 2015

DOI: $10.3892 /$ or.2015.4505

\begin{abstract}
Testicular germ cell tumors (TGCT) represent the most common malignant tumor group in the age group of 20 to 40 -years old men. The potentially curable effect of cytotoxic therapy in TGCT is mediated mainly by the induction of apoptosis. Autophagy has been discussed as an alternative mechanism of cell death but also of treatment resistance in various types of tumors. However, in TGCT the expression and role of core autophagy-associated factors is hitherto unknown. We designed the study in order to evaluate the potential role of autophagy-associated factors in the development and progression of testicular cancers. Eighty-four patients were assessed for autophagy (BAG3, p62) and apoptosis (cleaved caspase 3) markers using immunohistochemistry (IHC) on tissue microarrays. In addition, western blot analyses of frozen tissue of seminoma and non-seminoma were performed. Our findings show that BAG3 was significantly upregulated in seminoma as compared to non-seminoma but not to normal testicular tissue. No significant difference of p62 expression was detected between neoplastic and normal tissue or between seminoma and non-seminoma. BAG3 and p62 showed distinct loco-regional expression patterns in normal and neoplastic human testicular tissues. In contrast to the autophagic markers, apoptosis rate was significantly higher in testicular tumors as compared to normal testicular tissue, but not between different TGCT subtypes. The present study, for the first time, examined the expression of central autophagy proteins BAG3 and p62 in testicular cancer. Our findings imply that in general
\end{abstract}

Correspondence to: Dr Jens Mani, Department of Urology, Goethe University Frankfurt/Main, Theodor-Stern-Kai 7, D-60590 Frankfurt/Main, Germany

E-mail: jens.mani@kgu.de

${ }^{*}$ Contributed equally

Key words: autophagy, p62, BAG3, apoptosis, loco-regional pattern, testicular cancer apoptosis but not autophagy induction differs between normal and neoplastic testis tissue.

\section{Introduction}

The effect of cytotoxic treatment in testicular tumors is mainly moderated by the induction of apoptosis (1). Due to the rapid response after exposure to chemotherapeutic agents an intact and effective apoptotic pathway in most testicular cancers is assumed (2). Nevertheless, various patients show complex clinical courses, including tumors with chemoresistence and thus lower apoptosis rates (3). Recent studies have shown that the elimination of cells may be also conducted using the alternative cell death pathway 'autophagy' $(4,5)$.

Autophagy is an evolutionarily conserved, pervasive and multi-step 'self-eating' process, by which cytosolic material is sequestered in a double-lipid membrane, delivered to the lysosome for degradation and digested to provide energy and to build the foundation for cell-survival (6). While autophagy has for long been considered to be a bulk degradation process, recent discoveries show that it rather displays a sophisticated portfolio of selectivity provided by different molecular strategies such as the detection of several autophagy receptor proteins, including pacemaker molecules such as p62 (SQSTM1) and BCL2-associated athanogene 3 (BAG3) (7-9). To the best of our knowledge, no study has been published to date that examined the expression of the cohesive autophagy proteins BAG3 and p62 in testicular cancer. The aim of our study was to get first evidence for the potential role of autophagy-associated factors in the development and progression of testicular cancers. Therefore, we performed immunohistochemistry and western blot analyses for p62 and BAG3 of testicular cancer specimens of different entities and corresponding normal appearing testis tissue.

\section{Materials and methods}

Patient selection and clinicopathological analysis. Orchiectomy specimens of 84 testicular cancer patients from the Goethe University Hospital Frankfurt, Germany from 2002 to 2011 were evaluated. All patients provided written informed consent for the use of their tissues, and the study was approved by the Ethics Committee of the Goethe University 
Frankfurt/Main, Germany. All patients revealed adequate clinical follow-up data.

Tissue microarray. All tissue samples were retrieved from orchiectomy specimens, fixed in $10 \%$ buffered formalin and embedded in paraffin at time of surgery. For tissue microarray (TMA) construction hematoxylin and eosin-stained slides of human testicular germ cell tumors (TGCTs) were reviewed by a certified pathologist. The most representative areas of each tumor sample, and assigned corresponding biopsies of the transitional zone and the unaffected sites of the orchiectomy specimens, were marked and adequate cores were removed from the formalin-fixed paraffin-embedded (FFPE) block in triplicates. These samples were assembled in the array. The TMA location number was linked to the database including the clinicopathological data.

Immunohistochemistry. An automated immunostainer (Ventana, Strasbourg, France) using standard protocols was used for immunohistochemical staining. Briefly, 4- $\mu \mathrm{m}$ samples were heated to $100^{\circ} \mathrm{C}$, incubated with Inhibitor D (Ventana), and then incubated with the primary antibody (antibodies: p62, monoclonal mouse anti-p62 Ick ligand; dilution: 1:100; BD Transduction Laboratories, Franklin Lakes, NJ, USA; BAG3, polyclonal rabbit antibody; dilution, 1:100; Abnova, Taipei City, Taiwan; cleaved caspase 3, polyclonal rabbit antibody; dilution: 1:100; Cell Signaling, Danvers, MA, USA). The secondary antibody solution was incubated after rinsing, followed by sequential incubation with Blocker D and SA-HRP D (both from Ventana). Visualization was accomplished using diaminobenzidine (DAB) D and DAB $\mathrm{H}_{2} \mathrm{O}_{2} \mathrm{D}$ (Ventana). Finally, the slides were counterstained with hemalaun and mounted. The detailed immunohistochemical staining procedure was previously described (10). Notably, not every core of the TMA was evaluable for each protein (p62/BAG3) due to technical reasons, resulting in variations of numbers of the analyzed tissue specimens.

Scoring. The stained TMAs were evaluated with an Olympus BX50 light microscope. For semi-quantitative evaluation of the slides, a multi-score of staining frequency and intensity was applied. Staining frequency was assessed as follows: $0(0-1 \%), 1(1-10 \%), 2(10-25 \%), 3(25-50 \%)$, and $4(>50 \%)$. The staining intensity was rated as follows: no staining, 0 ; weak, 1 ; moderate, 2 ; and strong, 3 . This scoring system was previously described (11).

SDS-PAGE and western blotting. Frozen tissue of 3 tumors were available for analysis. Tumor and normal tissue were snap-frozen in liquid nitrogen, homogenized and incubated in lysis buffer (20 mM Tris- $\mathrm{HCl}$ buffer, pH 7.4; $150 \mathrm{mM} \mathrm{NaCl}$; $1.5 \mathrm{mM}$ ethylenediaminetetraacetic acid (EDTA); $3 \%$ glycerol; $1 \mathrm{mg} / \mathrm{ml} \mathrm{BSA} ; 1 \% \mathrm{NP}-40 ; 1 \mathrm{mg} / \mathrm{ml}$ leupeptin; $5 \mathrm{mg} / \mathrm{ml}$ aprotinin; $2 \mathrm{mg} / \mathrm{ml}$ pepstatin A) for $60 \mathrm{~min}$ at $0^{\circ} \mathrm{C}$. Protein lysates were loaded onto 12 or $15 \%$ SDS-polyacrylamide gels. Proteins were separated at $135 \mathrm{~V}$ and then blotted to nitrocellulose membranes (Protran BA83; 2 ml; Schleicher und Schuell, Dassel, Germany) in Towbin buffer [25 mM Tris, $192 \mathrm{mM}$ glycine and $20 \%$ methanol $(\mathrm{v} / \mathrm{v})]$ at $15 \mathrm{~V}$ for $35 \mathrm{~min}$. The blots were blocked in blocking buffer (5\% BSA, $50 \mathrm{mM}$
Tris- $\mathrm{HCl} \mathrm{pH} \mathrm{7.5,} 150 \mathrm{mM} \mathrm{NaCl}$ and $0.05 \%$ Tween-20) at $20^{\circ} \mathrm{C}$ for $1 \mathrm{~h}$. The resulting blots were probed with a rabbit monoclonal anti-BAG3 antibody diluted 1:3,000 (Biozol, Abnova), a mouse monoclonal anti-p62 antibody diluted 1:1,000 (BD Biosciences), a rabbit monoclonal anti-caspase 3 antibody diluted 1:1,000 (Cell Signaling) and a mouse monoclonal anti-glyceraldehyde-3-phosphate dehydrogenase (GAPDH) antibody (Calbiochem) diluted 1:10,000.

Statistical analysis. The semi-quantitative scores were assigned as ordinal scale response variable and analyzed along with nominal variables (tissue/tumor type). Non-parametric Wilcoxon test was used for statistical analyses. Corresponding normal and tumor tissues were assessed using a matched-pairs analyses. A significance level $\alpha=0.05$ was selected for all tests. Statistical analysis was performed using JMP 11.0.0 software (SAS, Cary, NC, USA).

\section{Results}

BAG3, but not p62, is significantly upregulated in seminoma as compared to both non-seminoma and normal testicular tissue. Immunohistochemical analyses for p62 (Fig. 1A-C) revealed that $\mathrm{p} 62$ protein was mainly detected within the parenchyma of normal testicular tissue (Fig. 1A), which was resected adjacent to non-seminomas (Fig. 1B) or classical seminomas (Fig. 1C). Notably, in both normal (Fig. 1A) and neoplastic (Fig. 1B and C) testicular tissue, stromal tissue components remained largely negative for $\mathrm{p} 62$. In general staining scores for $\mathrm{p} 62$ were rather low reaching a median expression score of 3 in both normal (range 1-6) and neoplastic (range 0-9) testicular tissue.

Only slightly higher expression values were obtained for BAG3. While normal testicular tissue (Fig. 1D) displayed a median expression score of 4 (range 0-9), non-seminomas (Fig. 1E) and seminomas (Fig. 1F) showed a median expression score of 3.5 (range 0-9) and 6 (range 1-12), respectively. In contrast to p62, BAG3 was also weakly expressed in stromal tissue of both normal (Fig. 1D) and neoplastic (Fig. 1E and F) specimens.

Statistical analyses of the immunohistochemical staining revealed no significant differences in p62 expression between normal and neoplastic testicular tissue (Fig. 2A) or between non-seminoma and seminoma (Fig. 2B).

While no significant differences were detected for BAG3, expression in normal testicular tissues when compared to testicular tumor tissues in general (Fig. 2C), seminomas displayed significantly higher BAG3 expression levels $(\mathrm{p}=0.0021)$ when compared to their non-seminomatous counterparts (Fig. 2D).

A matched-pairs analysis for p62 and BAG3 expression in non-neoplastic testicular tissues and their corresponding adjacent neoplastic tissues did not reveal any significant differences between the tissues (data not shown).

In contrast to the autophagy markers investigated in our study, apoptosis rates were significantly higher in testicular tumors as compared to tumor-adjacent normal testicular tissue while no differences in the apoptosis levels were seen in the comparison between seminomas and non-seminomas (Fig. 3). It is worthwhile mentioning that all investigated testicular tumors were chemonaive indicating that the detected apoptosis 

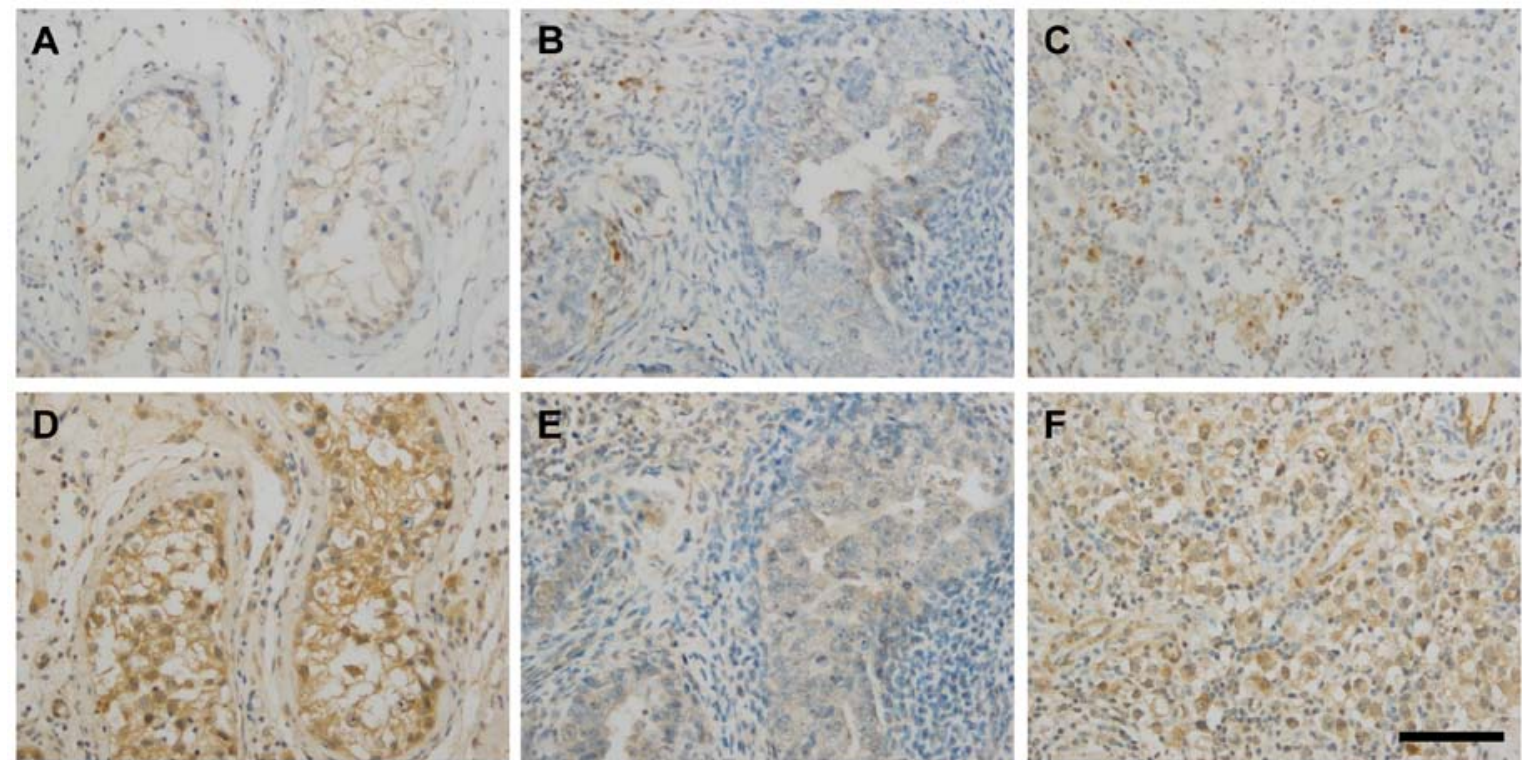

Figure 1. Immunohistochemistry of autophagy-associated molecules in human testicular cancer. p62 immunohistochemistry in (A) normal testis tissue, (B) non-seminoma (mixed small cell and embryonal carcinoma) and (C) classical seminoma. BAG3 immunohistochemistry in (D) normal testis tissue, (E) non-seminoma (mixed small cell and embryonal carcinoma) and (F) classical seminoma. Scale bar, $100 \mu$ m; magnification, x20 for all images.

A

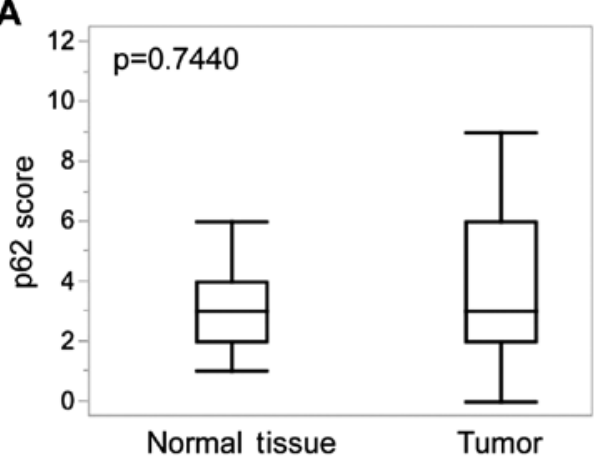

C

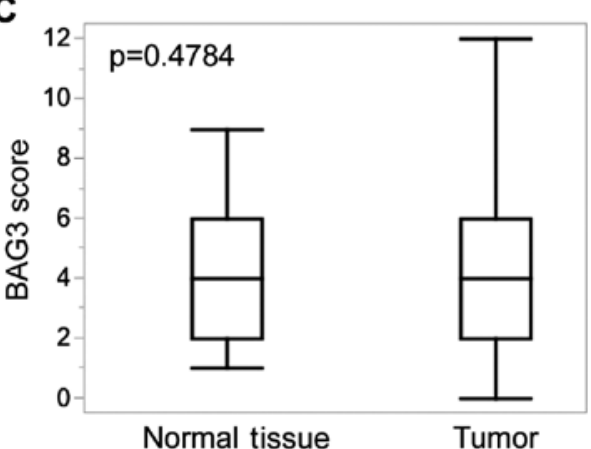

B

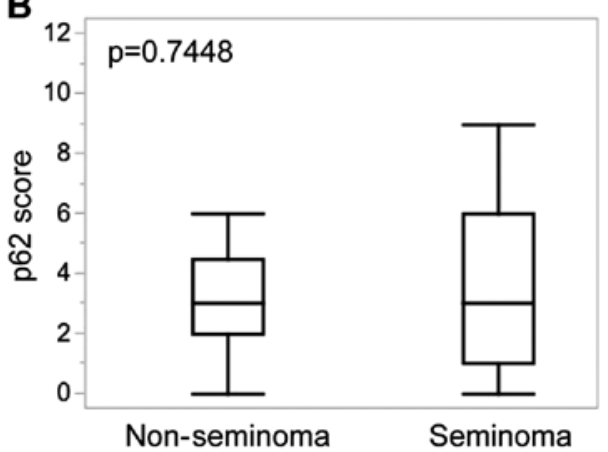

D

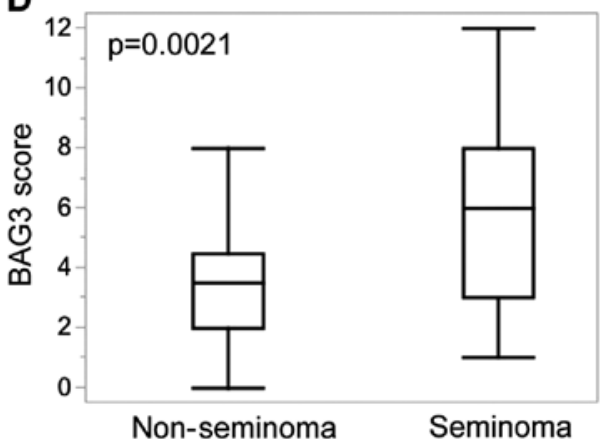

Figure 2. Statistical analysis of immunohistochemical stainings of TMA. Immunohistochemical staining scores are depicted for (A) p62 expression in 19 punches of normal appearing testicular tissue (median, 3; range, 1-6) and 66 punches of tumor tissue (median, 3; range; 0-9). (B) p62 expression in tumor tissue punches of 34 non-seminoma (median, 3; range, 0-9) and 32 seminoma specimens (median; 3; range, 0-9). (C) BAG3 expression in 23 normal appearing testicular tissue (median, 4; range, 0-9) and 73 punches of tumor tissue (median, 4; range, 0-12). (D) BAG3 expression in tumor tissue punches of 42 nonseminoma (median, 3.5; range, 0-9) and 31 seminoma specimens (median, 6; range, 1-12). p-values were obtained using non-parametric Wilcoxon test.

levels in our cohort reflect the endogenous rate of type-I cell death. Western blot analysis demonstrated that in contrast to unequivocally increased apoptosis levels in TGCT tissues, similarly or even slightly higher expression levels of BAG3 and/or p62 were detected in normal testicular control tissue as compared to corresponding TGCT samples (Fig. 4). p62 and BAG3 display distinct loco-regional expression patterns in normal and neoplastic human testicular tissues. Although, except for BAG3 expression in seminomatous vs. non-seminomatous tumors, statistical comparisons for p62 or BAG3 expression did not reveal significant differences between normal and neoplastic testicular tissues or between 

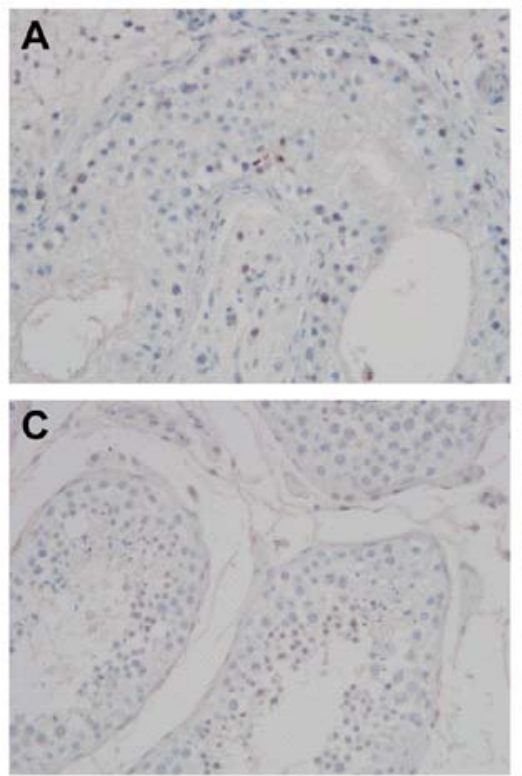
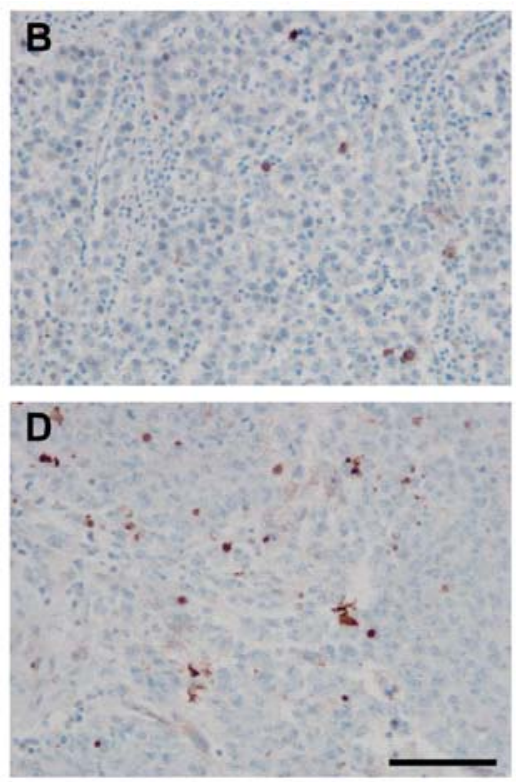
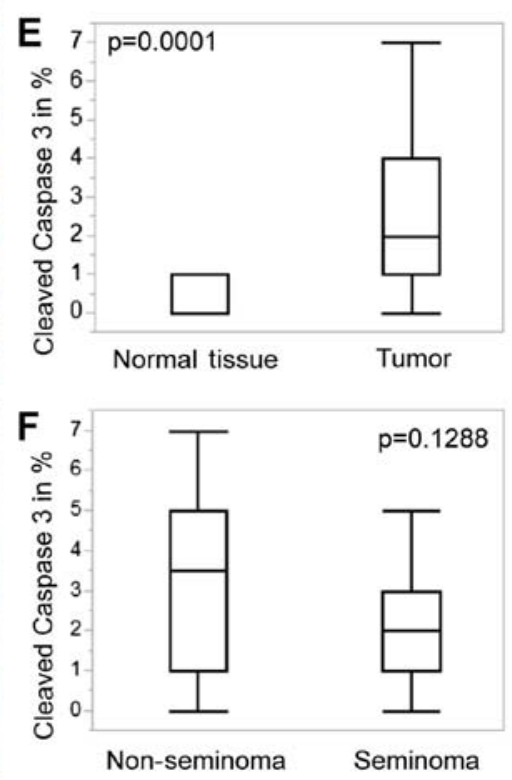

Figure 3. Cleaved caspase 3 expression in testicular cancer. (A) Cleaved caspase 3 immunohistochemistry in normal appearing testicular tissue (seminoma patient). (B) Cleaved caspase 3 immunohistochemistry in a seminoma (A and B, tissue from the same patient). (C) Cleaved caspase 3 immunohistochemistry in normal appearing testicular tissue (non-seminoma patient). (D) Cleaved caspase 3 immunohistochemistry, empryonal carcinoma (C and D, tissue from the same patient) (scale bar, $100 \mu \mathrm{m}$ for all images). (E) Cleaved caspase 3-positive cells in \% in 21 punches of normal appearing testis tissue (mean, $1 \%$; range $0-1 \%$ ) and 50 punches of tumor tissue (median, $2 \%$; range, $0-7 \%$ ). (F) Cleaved caspase 3 -positive cells in $\%$ in 20 punches of non-seminomas (median, $3.5 \%$; range, $0-7 \%$ ) and 30 seminomas (median, 2\%; range, 0-7\%); p-values were obtained using non-parametric Wilcoxon test.

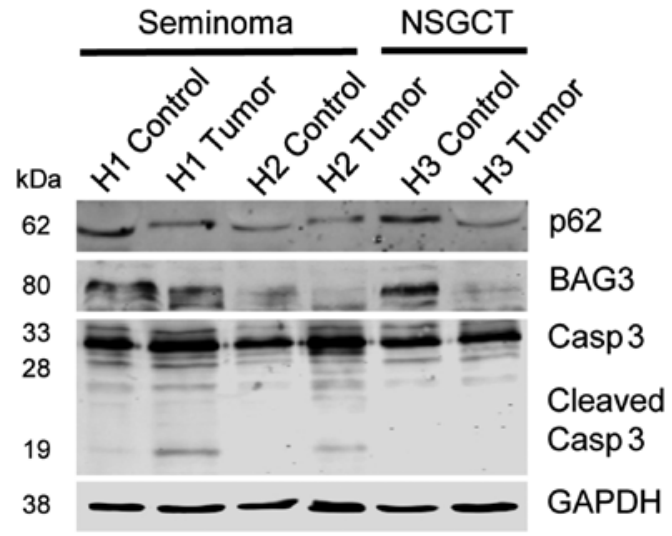

Figure 4. Protein biochemical analyses of autophagy- and apoptosis-associated molecules in normal and neoplastic testicular tissues. Western blot analysis of p62, BAG3 and cleaved caspase 3. GAPDH served as a loading control. $\mathrm{H} 1$ is a specimen of a seminoma pT2, $\mathrm{L} 1, \mathrm{~V} 0$ and $\mathrm{R} 0 . \mathrm{H} 2$ represents tissue of a seminoma pT3, L1, V0 and R0. H3 is an NSGCT, mixed malignant germ cell tumor consisting of a malignant teratoma ( $>50 \%)$, embryonic carcinoma $(\sim 30 \%)$ with a yolk sac tumor component, pT2, L1, V1 and R0.

the distinct seminomatous and non-seminomatous tumors, we aimed at taking a closer look at the immunohistochemically stained specimens with regard to potential expression differences in areas with distinct microenvironmental properties. In some perivascular areas in seminomas, both p62 (Fig. 5A and Aa) and BAG3 (Fig. 5B and Ba) expression was considerably stronger as compared to more remote tumor regions (Fig. 5Ab and $\mathrm{Bb}$ ).

Another repeatedly observed distinct staining pattern was found in perinecrotic areas of higher malignant testicular tumors such as embryonal carcinomas (Fig. 5C and D).
Both p62 (Fig. 5C) and BAG3 (Fig. 5D) were considerably upregulated in close proximity to necrotic foci, however, decreasing again at a distance of only a few micrometers from the border of the necrotic zone.

Finally, a slightly stronger protein expression of $\mathrm{p} 62$ (Fig. 5E) and BAG3 (Fig. 5F) was observed at the border zone between normal and neoplastic testicular tissue. Although the immunohistochemical results did not reach significance when the whole specimens were taken into account, these loco-regional staining differences may provide a more sophisticated view on autophagy-associated molecules in normal and neoplastic testicular tissues.

\section{Discussion}

Autophagy is highly contextual in regard to tumor cell death and survival decisions. In general autophagy acts as a prosurvival stress response, but it can also trigger cytodestructing effects (12-15). In our study we investigated the expression profiles of BAG3 and p62 in different types of testicular cancer and normal appearing testis tissue to gain insight into a potential in vivo relevance of autophagy in the development and progression of testicular cancer. Both p62 and BAG3 play a crucial role in the sophisticated portfolio of autophagy (16).

BAG3 was shown to sustain cell survival and it underlies resistance mechanisms to chemotherapy in several human neoplasms in vitro (17-19). Protein homeostasis in aged (or stressed) cells is driven by an increased BAG3 expression, regulated by the transcription factor heat shock factor 1 (HSF1) (20) and stabilized by the recruitment of the macroautophagy pathway involving HSP70, SQSTM1/p62 and LC3 $(7,21,22)$. It is also known that BAG3 mitigates proteotoxicity via selective autophagy (23). 

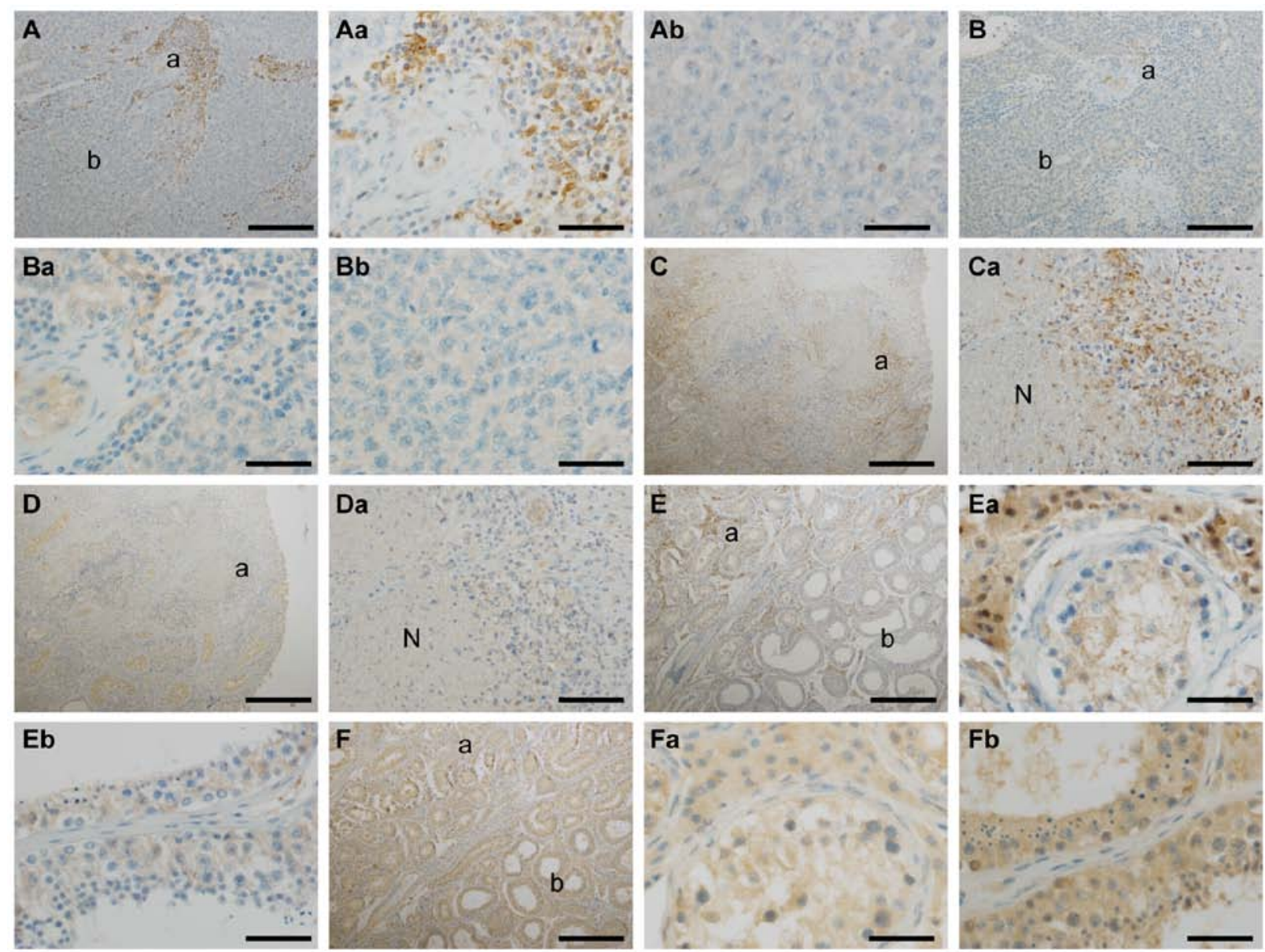

Figure 5. p62 and BAG3 protein expression display distinct loco-regional patterns. (A) p62 immunohistochemistry, seminoma, scale bar, $200 \mu \mathrm{m}$; (a) location of the zoomed-in image (Aa); (b) location of the zoomed-in image (Ab); (Aa) cut-out of (A), scale bar, $50 \mu \mathrm{m}$; (Ab) cut-out of (A), scale bar, $50 \mu \mathrm{m}$. (B) BAG3 immunohistochemistry, seminoma, scale bar, $200 \mu \mathrm{m}$; (a) location of the zoomed-in image (Ba); (b) location of the zoomed-in image (Bb); (Ba) cut-out of (B), scale bar, $50 \mu \mathrm{m}$; (Bb) cut-out of (B), scale bar, $50 \mu \mathrm{m}$. (C) p62 immunohistochemistry, testicular embryonal carcinoma with necrosis; (a) location of the zoomed-in image (Ca), scale bar, $500 \mu \mathrm{m}$; (Ca) cut-out of (C), 'N' indicating necrosis, scale bar, $100 \mu \mathrm{m}$. (D) BAG3 immunohistochemistry, testicular embryonal carcinoma; (a) location of the zoomed-in image (Da), scale bar: $500 \mu \mathrm{m}$; (Da) cut-out of (D), 'N' indicating necrosis, scale bar, $100 \mu \mathrm{m}$. (E) p62 immunohistochemistry, overview of a border zone of a seminoma, scale bar, $500 \mu \mathrm{m}$; (a) ocation of the zoomed-in image (Ea); (b) location of the zoomed-in image (Eb); (Ea) cut-out of (E), scale bar, $50 \mu \mathrm{m}$; (Eb) cut-out of (E), scale bar, $50 \mu \mathrm{m}$. (F) BAG3 immunohistochemistry, overview of a border zone of a seminoma, scale bar, $500 \mu \mathrm{m}$; (a) location of the zoomed-in image (Fa); (b) location of the zoomed-in image (Fb); (Fa) cut-out of (F), scale bar, $50 \mu \mathrm{m}$; (Fb) cut-out of $(\mathrm{F})$, scale bar, $50 \mu \mathrm{m}$.

The cargo receptor p62 functionally interfaces to the autophagic machinery through degradation of ubiquitin-tagged proteins. p62 recruits protein aggregates as substrate to autophagic membranes to form autophagosomes and thus promotes their degradation (24). In addition, p62 itself is a substrate for autophagic degradation and accumulates upon inhibition of autophagy $(24,25)$.

We noted that BAG3 and p62 are not upregulated in testicular cancers as compared to normal testicular tissue in both immunohistochemical and immunoblotting analyses. The normal tissue even shows slightly higher expression levels of BAG3 and/or p62. The expansive growing tumor may induce pressure on the normal tissue particularly in a cloistered organ such as the testis. The resulting insufficient supply of e.g. oxygen and changes in microenvironmental nutrition may induce autophagy in order to mitigate the adverse condition and then avoid cell damage or even cell death (26). Our findings implicate that microscopically normal appearing testicular tissue may be stressed at the molecular level due to the neighboring pathologies and thus induces protective mechanisms.

In regards to BAG3 these results are contrary to previous studies which detected substantial BAG3 expression in several types of human cancers, such as glioblastoma, pancreatic carcinoma, leukemia and thyroid carcinoma, compared with very low basal levels of BAG3 in non-malignant cells $(18,27,28)$. Other studies have reported that an increased expression of BAG3 was shown to induce the formation of p62 bodies (9) and thus promoting selective autophagy via its interaction with p62. In our case, p62 may not be upregulated, hypothetically due to the fact that p62 itself is a substrate for autophagic degradation (9). We observed that in both normal and neoplastic testicular tissue, stromal tissue components remained largely negative for p62. In contrast, previous studies have shown that stromal p62 expression was associated with the histological grade and molecular subtype (29,30). Our results demonstrated that there is no significant difference in autophagy induction in neoplastic tissue when compared to normal testicular tissue. 
Contrary to these findings, apoptotic activity is significantly increased in testicular tumor, but not in normal testicular tissue, as proliferative tumor tissue of a certain size usually becomes hypoxic, eventually necrotic and thus enhanced apoptosis can be detected particularly at the margins of hypoxic or necrotic tumor areas $(31,32)$. Up to now the loco-regional expression patterns of autophagy in TGCTs have not been a subject of research. We believe that a 'topographical' point-of-view may be a determinant for the interpretation of our results. In our case, areas of crucial relevance for local and systemic tumor progression and survival show increased expression patterns of p62 and BAG3. When having a holistic view on the staining a slightly stronger protein expression for p62 and BAG3 was noted at the border zone where tumor pins protrude into the normal testicular tissue, a fact that may represent a protective barrier, in order to protect the tumor.

Another repeatedly observed distinct staining pattern of BAG3 and p62 was found in perinecrotic (where presumably hypoxia is maximal) areas of higher malignant testicular tumors such as embryonal carcinomas. Autophagy induction in cancer cells may occur due to hypoxia $(13,32)$, and it has been previously demonstrated that expression of hypoxiainducible factor $1 \alpha$ (HIF-1 $\alpha)$ downstream targets is associated with a higher histologic grade in fibroepithelial tumors of the breast (33). In this scenario autophagy provides the nutrients needed for cancer survival and stabilization of HIF-1 $\alpha$ allows for instance that higher grade phyllode tumors adapt to hypoxia (30). In another clinical setting, the cardiac infarction, this hypothesis has been verified. Autophagy is more prominent in cardiomyocytes in the border zone than in those in more remote areas, and the size of the autophagosomes was exceedingly large in the former. Previously, Kanamori et al suggested a protective role during post-infarction cardiac remodeling (34). Surprisingly contrary to these findings, in some perivascular areas in seminoma, presumably a site with optimal oxygenation and nutrient status, both p62 and BAG3 expression was considerably stronger as compared to more remote tumor regions.

Up to now there are no studies that have explored the relationship between seminoma and non-seminoma in respect to autophagy. We observed that BAG3 is significantly upregulated in seminoma compared to non-seminoma. This difference may be due to the heterogeneity of testicular germ cell tumors (TGCT). As stated by Elstrand et al, discrepancies may be due to the very complex types of intracellular molecular interaction of the BAG3 proteins (i.e. with HSP proteins and $\mathrm{Bcl}-2$ members) in tumors of various origin and differentiation $(22,27,35,36)$. While seminoma and non-seminoma show great similarity in etiology, precursor lesions and chromosome $12 p$ alterations, different TGCT subtypes display distinct tumor genetics that may explain their different histology and biological behavior. Studies have revealed differences between seminoma and nonseminoma e.g. for KRAS, NRAS and BRAF (37). Different genetic factors may also influence the autophagic behavior of the testicular tumor entities. Further investigations are necessary to determine the exact expression profile of autophagy proteins in different types of testicular cancer.

The limitations of the present study are defined through the fact that autophagy is a dynamic, multistep process and the accurate measurement of autophagy involves the quantification of autophagic flux (38). Therefore, the identification of proteins associated with autophagy or those that are degraded by autophagy through quantitative methods such as immunohistochemistry (IHC)/tissue microarray (TMA) may be problematic. These methods provide a snapshot of the identified proteins at a specific point in time and are actually unable to reflect the activity of autophagy.

In conclusion, even though autophagy markers display a loco-regional expression pattern in normal and neoplastic human testicular tissue, our findings imply that in general apoptosis, but not autophagy induction differs between normal and neoplastic testis tissue. The precise differences and interrelationships of autophagy in respect to seminoma and non-seminoma have to be further investigated with an autophagy-related molecular calibration of these two distinct types of testicular cancer.

\section{References}

1. Mayer F, Stoop H, Scheffer GL, Scheper R, Oosterhuis JW, Looijenga LH and Bokemeyer C: Molecular determinants of treatment response in human germ cell tumors. Clin Cancer Res 9: 767-773, 2003.

2. Spierings DC, de Vries EG, Vellenga E and de Jong S: The attractive Achilles heel of germ cell tumours: An inherent sensitivity to apoptosis-inducing stimuli. J Pathol 200: 137-148, 2003.

3. Hanahan D and Weinberg RA: Hallmarks of cancer: The next generation. Cell 144: 646-674, 2011.

4. Lian J, Wu X, He F, Karnak D, Tang W, Meng Y, Xiang D, Ji M, Lawrence TS and Xu L: A natural BH3 mimetic induces autophagy in apoptosis-resistant prostate cancer via modulating Bcl-2-Beclin1 interaction at endoplasmic reticulum. Cell Death Differ 18: 60-71, 2011.

5. Voss V, Senft C, Lang V, Ronellenfitsch MW, Steinbach JP, Seifert V and Kögel D: The pan-Bcl-2 inhibitor (-)-gossypol triggers autophagic cell death in malignant glioma. Mol Cancer Res 8: 1002-1016, 2010.

6. Nakatogawa H, Suzuki K, Kamada Y and Ohsumi Y: Dynamics and diversity in autophagy mechanisms: Lessons from yeast. Nat Rev Mol Cell Biol 10: 458-467, 2009.

7. Mizushima N, Levine B, Cuervo AM and Klionsky DJ: Autophagy fights disease through cellular self-digestion. Nature 451: 1069-1075, 2008.

8. Amaravadi RK, Lippincott-Schwartz J, Yin XM, Weiss WA, Takebe N, Timmer W, DiPaola RS, Lotze MT and White E: Principles and current strategies for targeting autophagy for cancer treatment. Clin Cancer Res 17: 654-666, 2011.

9. Gamerdinger M, Hajieva P, Kaya AM, Wolfrum U, Hartl FU and Behl C: Protein quality control during aging involves recruitment of the macroautophagy pathway by BAG3. EMBO J 28: 889-901, 2009.

10. Baumgarten P, Harter PN, Tönjes M, Capper D, Blank AE, Sahm F, von Deimling A, Kolluru V, Schwamb B, Rabenhorst U, et al: Loss of FUBPI expression in gliomas predicts FUBP1 mutation and is associated with oligodendroglial differentiation, IDHI mutation and $1 \mathrm{p} / 19 \mathrm{q}$ loss of heterozygosity. Neuropathol Appl Neurobiol 40: 205-216, 2014.

11. Harter PN, Bunz B, Dietz K, Hoffmann K, Meyermann R and Mittelbronn M: Spatio-temporal deleted in colorectal cancer (DCC) and netrin-1 expression in human foetal brain development. Neuropathol Appl Neurobiol 36: 623-635, 2010.

12. Baehrecke EH: Autophagy: Dual roles in life and death? Nat Rev Mol Cell Biol 6: 505-510, 2005.

13. Degenhardt K, Mathew R, Beaudoin B, Bray K, Anderson D, Chen G, Mukherjee C, Shi Y, Gélinas C, Fan Y, et al: Autophagy promotes tumor cell survival and restricts necrosis, inflammation, and tumorigenesis. Cancer Cell 10: 51-64, 2006.

14. Roy S and Debnath J: Autophagy and tumorigenesis. Semin Immunopathol 32: 383-396, 2010.

15. Galluzzi L, Pietrocola F, Bravo-San Pedro JM, Amaravadi RK, Baehrecke EH, Cecconi F, Codogno P, Debnath J, Gewirtz DA, Karantza V, et al: Autophagy in malignant transformation and cancer progression. EMBO J 34: 856-880, 2015. 
16. Johansen T and Lamark T: Selective autophagy mediated by autophagic adapter proteins. Autophagy 7: 279-296, 2011.

17. Chen HY, Liu P, Sun M, Wu LY, Zhu HY, Qiao C, Dong HJ, Zhu DX, Xu W and Li JY: Bag3 gene expression in chronic lymphocytic leukemia and its association with patients prognosis. Zhongguo Shi Yan Xue Ye Xue Za Zhi 18: 838-842, 2010 (In Chinese).

18. Liao Q, Ozawa F, Friess H, Zimmermann A, Takayama S, Reed JC, Kleeff J and Büchler MW: The anti-apoptotic protein BAG-3 is overexpressed in pancreatic cancer and induced by heat stress in pancreatic cancer cell lines. FEBS Lett 503: 151-157, 2001.

19. Boiani M, Daniel C, Liu X, Hogarty MD and Marnett LJ: The stress protein BAG3 stabilizes Mcl-1 protein and promotes survival of cancer cells and resistance to antagonist ABT-737. J Biol Chem 288: 6980-6990, 2013.

20. Rosati A, Ammirante M, Gentilella A, Basile A, Festa M, Pascale M, Marzullo L, Belisario MA, Tosco A, Franceschelli S, et al: Apoptosis inhibition in cancer cells: A novel molecular pathway that involves BAG3 protein. Int J Biochem Cell Biol 39: 1337-1342, 2007.

21. Behl C: BAG3 and friends: Co-chaperones in selective autophagy during aging and disease. Autophagy 7: 795-798, 2011.

22. Li X, Colvin T, Rauch JN, Acosta-Alvear D, Kampmann M, Dunyak B, Hann B, Aftab BT, Murnane M, Cho M, et al: Validation of the Hsp70-Bag3 protein-protein interaction as a potential therapeutic target in cancer. Mol Cancer Ther 14: 642-648, 2015.

23. Rapino F, Jung M and Fulda S: BAG3 induction is required to mitigate proteotoxicity via selective autophagy following inhibition of constitutive protein degradation pathways. Oncogene 33: 1713-1724, 2014.

24. Yao TP: The role of ubiquitin in autophagy-dependent protein aggregate processing. Genes Cancer 1: 779-786, 2010.

25. Hua F, Li K, Yu JJ, Lv XX, Yan J, Zhang XW, Sun W, Lin H, Shang S, Wang F, et al: TRB3 links insulin/IGF to tumour promotion by interacting with p62 and impeding autophagic/proteasomal degradations. Nat Commun 6: 7951, 2015.

26. Filomeni G, De Zio D and Cecconi F: Oxidative stress and autophagy: The clash between damage and metabolic needs. Cell Death Differ 22: 377-388, 2015.

27. Romano MF, Festa M, Petrella A, Rosati A, Pascale M, Bisogni R, Poggi V, Kohn EC, Venuta S, Turco MC, et al: BAG3 protein regulates cell survival in childhood acute lymphoblastic leukemia cells. Cancer Biol Ther 2: 508-510, 2003.
28. Festa M, Del Valle L, Khalili K, Franco R, Scognamiglio G, Graziano V, De Laurenzi V, Turco MC and Rosati A: BAG3 protein is overexpressed in human glioblastoma and is a potential target for therapy. Am J Pathol 178: 2504-2512, 2011.

29. Choi J, Jung W and Koo JS: Expression of autophagy-related markers beclin-1, light chain 3A, light chain 3B and p62 according to the molecular subtype of breast cancer. Histopathology 62 : 275-286, 2013.

30. Kim JY, Jung WH and Koo JS: Expression of autophagy-related proteins according to androgen receptor and HER-2 status in estrogen receptor-negative breast cancer. PLoS One 9: e105666, 2014.

31. Elmore S: Apoptosis: A review of programmed cell death. Toxicol Pathol 35: 495-516, 2007.

32. Qiu Y, Li P and Ji C: Cell death conversion under hypoxic condition in tumor development and therapy. Int J Mol Sci 16: 25536-25551, 2015.

33. Kuijper A, van der Groep P, van der Wall E and van Diest PJ: Expression of hypoxia-inducible factor 1 alpha and its downstream targets in fibroepithelial tumors of the breast. Breast Cancer Res 7: R808-R818, 2005.

34. Kanamori H, Takemura G, Goto K, Maruyama R, Tsujimoto A, Ogino A, Takeyama T, Kawaguchi T, Watanabe T, Fujiwara T, et al: The role of autophagy emerging in postinfarction cardiac remodelling. Cardiovasc Res 91: 330-339, 2011.

35. Elstrand MB, Kleinberg L, Kohn EC, Tropé CG and Davidson B: Expression and clinical role of antiapoptotic proteins of the bag, heat shock, and Bcl-2 families in effusions, primary tumors, and solid metastases in ovarian carcinoma. Int J Gynecol Pathol 28: 211-221, 2009.

36. Franceschelli S, Rosati A, Lerose R, De Nicola S, Turco MC and Pascale M: Bag3 gene expression is regulated by heat shock factor 1. J Cell Physiol 215: 575-577, 2008.

37. Sheikine Y, Genega E, Melamed J, Lee P, Reuter VE and Ye H: Molecular genetics of testicular germ cell tumors. Am J Cancer Res 2: 153-167, 2012.

38. Mizushima N, Yoshimori $\mathrm{T}$ and Levine B: Methods in mammalian autophagy research. Cell 140: 313-326, 2010. 